\title{
An Investigation of Mechanical Strength of Yarn or Cord Twisted with Other Kinds of Filaments
}

\author{
By Hideo Noshi, Teruo Ishida and Masanosuke Shimada, Members, TMSJ
}

\author{
Faculty of Technical Arts, Kyoto University of Technical Arts \\ and Textile Fibers, Kyoto
}

\begin{abstract}
The object of this study is to discover the most effective law of blending and the maximum limitation on the mechanical strength of a blended yarn or cord composed of two kinds of continuous filaments.

In the case of tensile rupture, the fibers making up a blended yarn resist the rupture most effectively if the mixing rate of the fibers of low extensibility is under $25 \%$ and the twist on the blended yarns is more than half the permissible maximum twist.

In the case of flexing fatigue, an ideal blended cord is obtainable if the parameter of S-N diagrams and the mechanical model of the constituent fibers of the blended cord satisfy equation (27).

The tensile strength or fatigue life of even the most effective blended yarn or cord is less than that of the mathematical mean of the constifuent fibers. The amount of work (the degree of toughness) needed for the tensile rupture of the yarn or cord exceeds the mathematical mean of the constituent fibers.
\end{abstract}

\section{Introduction}

The coming of an increasing variety of fibers has given birth to the science of blending and mixing. Many experimental results on the properties $[1] \sim[3]$ of blended or mixed products have been reported, but few adequate theoretical investigations have been made.

The authors have inquired theoretically and experimentally into the law of blending to increase the elongation and tensile strength of yarns or cords, each made of two kinds of continuous filaments, the work energy required for the tensile rupture of such yarns and cords and their fatigue strength. The purpose of the inquiry is to establish a basis for judging what kinds of fibers may be blended for use where mechanical strength is required.

\section{Tensile Rupture of Twist Yarn}

\section{1-1. Length of Fragments of Filaments $S_{1}$}

Assume two kinds of continous filaments drafted and twisted togather. $S_{2}$, one of the two kinds of filaments, has a larger breaking elongation than the other kind $S_{1}$. If a twisted yarn of a low twist degree is elongated to the breaking elongation point of $S_{1}$, then $S_{1}$ breaks into two fragments. If, however, a twisted yarn of a high twist degree is elongated to the same breaking elongation point, $S_{1}$, breaks into several fragments of different lengths $l_{r}$, depending on their distance from the center of the yarn. $l_{r}$ is calculable by Fujino's theory [4] on tensile strength of spun yarn. The following symbols are used:

$T_{1}$ : tension of the fragments of $S_{1}$.

$G$ : perpendicular force produced by $T_{1}$ (per unit length).

$K_{1}$ : adhesive force inherent in fibers.

$K_{2}$ : pressure from external filaments (per unit length).

$\rho$ : radius of curvature of twist spiral.

$S$ : length along the filament.

$\mu$ : inter-filament frictional coefficient.

$M$ : number of twists per unit length.

$\sigma_{1 b}$ : stress required for the rupture of filaments.

$L$ : length of filaments constituting the sample ( $\fallingdotseq$ test length of sample yarn).

$f_{A}(\varepsilon)$ : stress-strain curve of a twisted yarn composed only of $S_{1}$.

$f_{B}(\varepsilon)$ : stress-strain curve of a twisted yarn composed only of $S_{2}$.

The balance of the force of a small element dS of broken $S_{1}$ in a yarn during elongaion is deducible from the following equations (see also Fig, 1) :

$$
\begin{array}{r}
T_{1}+d T_{1}=T_{1}+\mu\left\{d G+2\left(K_{1}+K_{2}\right) d S\right\} \\
\therefore d T_{1}=\mu\left\{d G+2\left(K_{1}+K_{2}\right) d S\right\} \quad \ldots
\end{array}
$$


about $d G$

$$
\begin{aligned}
& d \phi=d S / \rho=d G / T_{1} \\
& \therefore d G=\left(T_{1} / \rho\right) d S
\end{aligned}
$$

Therefore, equation (2) transforms itself into:

$$
d T_{1}=(\mu / \rho) \cdot\left\{T_{1}+2\left(K_{1}+K_{2}\right) \rho\right\} d S
$$

Resolving this differential equation gives us the following equation:

$$
T_{1}=C \cdot e^{\langle\mu / \rho) \zeta S}-2\left(K_{1}+K_{2}\right) \rho
$$

Assuming $S=0, T$ is zero. Hence:

$$
\begin{aligned}
& C=2\left(K_{1}+K_{2}\right) \rho \\
\therefore & T_{1}=2\left(K_{1}+K_{2}\right) \rho\left\{e^{(\mu / \rho) S}-1\right\}
\end{aligned}
$$

Equation (5) represents the distribution of the tension of the fragments. $T_{1 \max }$ i. e., the maximum tension, develops in the center of a filament, i. e., at $l_{r} / 2$. Since $S_{\mathrm{I}}$ breaks when $T$ $r_{\max }=\sigma_{1 b}, l_{r}$ is:

$$
2\left(K_{1}+K_{2}\right) \rho\left\{e^{\langle\mu / \rho)\langle(t / 2)}-1\right\}=\sigma_{1 b} \quad \ldots \ldots
$$

To express $l_{r}$ as the function of the number $M$ of twists and as the function of the distance from the center $r$, we have to express $\rho, K_{1}$ and $K_{2}$ with $M$ and $r . \rho$ is expressed with $r$ and $M$ thus:

$$
\rho=\frac{1}{r}\left\{r^{2}+\frac{1}{(2 \pi M)^{2}}\right\}=\frac{1}{r(2 \pi M)^{2}}
$$

$K_{1}$ is not calculable. The larger $K_{2}$ is, the smaller $r$ is. $K_{2}$ is calculable. The filaments $S_{1}$ of a cord or rope made of multi-filament yarns break into fragments of length $l_{r}$, which is given by equation (6). Because all filaments seldom, if ever, break at the same point, the bunch of $S_{1}$ may be thought of as being in the same state as a spun yarn.

The theory of spun yarn is that filaments break if they are so long in length or the pressure on them is so high that their maximum tension $T_{1 \max }$ exceeds $\sigma_{1} b$, while they slip out if $T_{1 \max }$ is smaller than $\sigma_{1} b$. The force needed to break the filaments is $\sigma_{1} b$. The force needed to make them slip out is smaller than $\sigma_{1}$. However, since inter-filament pressure breaks $S_{1}$ in a length which satisfies $T_{1 \max }=\sigma_{1} b$, the force needed to

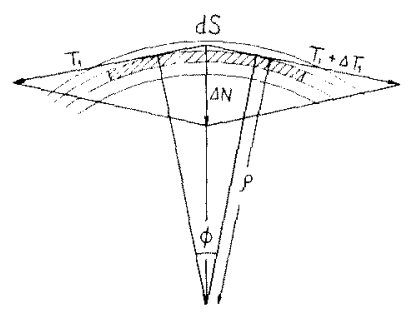

Fig. 1. Balance of tension on fiber. eject $S_{1}$ is $\sigma_{1 b}$.

If filaments $S_{1}$ of a yarn of a high twist degree break, the tensile stress of the yarn does not decrease by reason of the friction of fragments. If $S_{1}$ of a yarn of a low twist degree breaks, the tensile stress of the yarn does decrease to a certain extent because the frictional resistance of the external filaments is inadequate. Accordingly, tensile stress $\sigma_{t 3}$ of a twisted yarn just before its tensile strain equals the breaking elongation of $S_{1}$, and tensile stress $\sigma_{t 4}$ just before filament $S_{2}$ breaks, are given as follows:

$$
\begin{aligned}
\sigma_{t 3} & =A_{1} f_{A}\left(\varepsilon_{1 b}\right)+B_{1} f_{B}\left(\varepsilon_{1 b}\right) \\
& =A_{1}\left\{f_{A}\left(\varepsilon_{1 b}\right)-f_{B}\left(\varepsilon_{1 b}\right)\right\}+f_{B}\left(\varepsilon_{1 b}\right) \quad \ldots \\
\sigma_{i 4} & =B_{1} f_{H}\left(\varepsilon_{2 b}\right)+\alpha A_{1} f_{A}\left(\varepsilon_{1 b}\right) \quad \ldots \ldots
\end{aligned}
$$

where $0<\alpha<1, A_{1}$ and $B_{1}$ are fractions of $S_{1}$ and $S_{2}$, respectively, $\left(A_{1}+B_{1}=1\right) . \varepsilon_{1 b}$ and $\varepsilon_{2 b}$ are the breaking elongations of $S_{1}$ and $S_{2}$.

The stress-strain curve in this range is given by the following equation:

$$
\sigma=B_{1} f_{B}(\varepsilon)+\alpha A_{1} f_{A}(\varepsilon)
$$

When $\alpha$ nearly equals one, $\sigma_{t 4}$ equals the tensile strength of a blended yarn. $\alpha=1$ is the most effective blended and twisted yarn.

\section{1-2. The Lowest Degree of Twist for Making $\alpha=1$}

$\alpha$ depends on the degree of twist, frictional coefficient between filaments, the ratio of mixing, the condition of mixing and the tensile stress of filament $S_{2}$. Let us calculate the lowest degree of twist which can make $\alpha$ of a twist yarn equal one. For the sake simplification, we will assume an external phase completely covered with $S_{2}$ and a low $S_{1}$ content.

Assume $S_{01}$, the filament in the most external position of all $S_{1}$ filaments, ruptured by tension. If the frictional resistance of fragments of $S_{01}$ is brought into full view by pressure from $S_{2}$ which surround $S_{01}$, then the frictional resistance of the fragments of $S_{1}$ - a filament which is further inside than $S_{01}$ - is also brought into full view as a matter of course. Then $\alpha$ ought to equal one. For the fragments of $S_{1}$ to display full frictional resistance, there must be at least two fragments of complete length $l_{r}$ in $L$. Thus:

$$
2 l_{r} \leqq L
$$

The minimum twist needed for $\alpha=1$ near the cut-point of $S_{1}$, therefore, must satisfy $2 l_{0 r}=L$, where $l_{0 r}$ is the length of the fragments of $S_{01}$. Equation (6), then, transforms itself into:

$$
2\left(K_{1}+K_{2}\right) \rho_{0}\left\{e^{(\mu / \rho 0)(L / 4)}-1\right\}=\sigma_{1 b} \quad \ldots
$$


where $\rho_{0}$ is the curvature of $S_{01}$.

$T_{2}$, the tensile stress of $S_{2}$ when $S_{1}$ breaks, is $T_{2}=f_{2}\left(\varepsilon_{1} b\right)$, where $f_{2}(\varepsilon)$ is the stress-strain curve of $S_{2}$. From equations (3) and (7) emerges this further equation:

$$
K_{2}=T_{2} / \rho_{0} \fallingdotseq r_{0}(2 \pi M)^{2} f_{2}\left(\varepsilon_{1 b}\right) \quad \ldots
$$

where $r_{0}$ is the radius of the yarn.

Assuming $S_{1}$ to be covered with several phases of $S_{2}$ to the number of $i$, then $K_{2}$ is:

$$
K_{2}=i \cdot r_{i} \cdot f_{2}\left(\varepsilon_{1 b}\right)(2 \pi M)^{2}
$$

Assuming $K_{1}$ to be equal to zero, $M_{m i n}-$ the minimum number of twists needed for $\alpha=1$ -can be calculated as follows: From equations (12), (7) and (13):

$$
\begin{aligned}
& \left.2 i f_{2}\left(\varepsilon_{1 b}\right)\left[e^{\left\{\mu r_{i}\left(2 \pi M_{m i n}\right)^{2} L\right\}}\right\}^{4}-1\right]=\sigma_{1 b} \\
\therefore & M_{m i n}=\left[\frac{\log \left[\left\{\sigma_{1 b} / 2 i f_{2}\left(\varepsilon_{1 b}\right)\right\}+1\right]}{L \mu \gamma_{i} \pi^{2}}\right]^{1 / 2} \ldots
\end{aligned}
$$

$M_{m i n}$ depends on $L$. In other words, it is inversely proportional to $L^{1 / 2}$

1-3. Elongation and Work Energy Needed for Rupture When $\alpha=1$

Equation (9) shows that $\sigma_{l 4}$, the tensile strength when $\alpha=1$, is equal to the arithmetical mean of $f_{B}\left(\varepsilon_{2 b}\right)$ and $f_{A}\left(\varepsilon_{1 b}\right)$. The breaking elongation always exceeds $\varepsilon_{2} b$. Then $W_{t}$, the work energy needed for breaking, is:

$$
\begin{aligned}
W_{t}=A_{1} \int_{0}^{\varepsilon_{1 b}} f_{A}(\varepsilon) d \varepsilon+B_{1} \int_{0}^{\varepsilon_{2 b}} f_{B}(\varepsilon) d \varepsilon \\
+A_{1}\left\{f_{A}\left(\varepsilon_{1 b}\right)\right\}\left(\varepsilon_{2 b}-\varepsilon_{1 b}\right)
\end{aligned}
$$

$W_{l}$, therefore, exceeds, by the third term, the arithmetical mean of $f_{A}(\varepsilon)$ and $f_{B}(\varepsilon)$ at various ratios of mixing.

\section{1-4. Experiment on $\alpha$}

Although $\alpha$ increases with an increase in the number of twists, the relation between $\alpha$ and the number of twists varies with the mixing ratio

\begin{tabular}{|c|c|c|c|c|}
\hline Yarns & Den & $\begin{array}{l}\text { Tensile } \\
\text { strength } \\
\text { (g/den.) }\end{array}$ & $\begin{array}{c}\text { Elongation- } \\
\text { to-rupture } \\
(\% \delta)\end{array}$ & $\begin{array}{l}\text { Stress on } \\
23 \% \text { elonga- } \\
\text { tion g/den. }\end{array}$ \\
\hline Rayon & 120 & 1.75 & 23 & 1.75 \\
\hline Amilan & 110 & 3. 31 & 48 & 3.00 \\
\hline
\end{tabular}
of filaments. Rayon and Amilan filaments of the properties shown in Table 1 were twisted together in varying proportions of mixing and twisting -

Table 1. Tensile Properties of Tested Yarn $\left(20^{\circ} \mathrm{C}, 65 \%\right.$ R.H.) about 37 filaments in total being used in each instance and made into yarns of about 4,100 denier. Care was taken to make each mixing complete. The twist degree of each yarn and $\alpha$ were measured with a pendulum-type tensile tester at $20^{\circ} \mathrm{C}, 65 \% \mathrm{R} . \mathrm{H}$. to discover the relation between them. The relation is shown in Figure 2, which is based on twist coefficient $K$.

$$
\begin{gathered}
K=\sqrt{D} \cdot M / 1,000 \quad M: \text { number of twists } \\
\text { per meter } \\
D: \text { denier of yarn }
\end{gathered}
$$

$\alpha$ was calculated by the following equation:

$$
\alpha=1-\frac{W}{A_{1} f_{A}\left(\varepsilon_{1 b}\right)}
$$

$W$ is the decreased stress when $f_{A}\left(\varepsilon_{1 b}\right)$ breaks; $f_{A}\left(\varepsilon_{1} b\right)$, the tensile strength of viscose rayon in various degrees of twist.

$A$ yarn low in $S_{1}$ content reaches $\alpha=1$ with a small degree of twist. A yarn of a high $S_{1}$ content needs a high degree of twist to reach $\alpha=1$. A yarn in which the $S_{1}$ content exceeds some definite value does not reach $\alpha=1$. A yarn containing $10 \sim 20 \%$ viscose rayon was measured

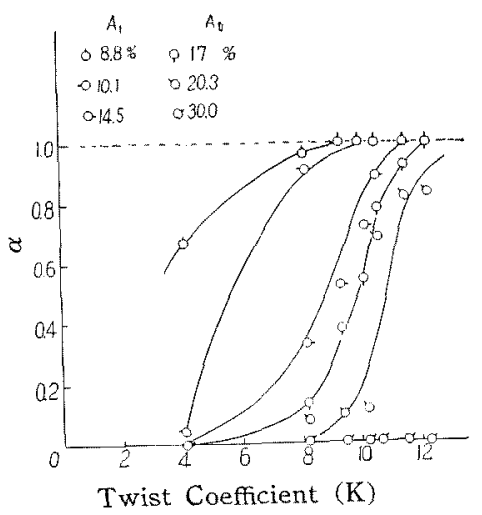

Fig. 2. Relation between $\alpha$ and twist coefficient.

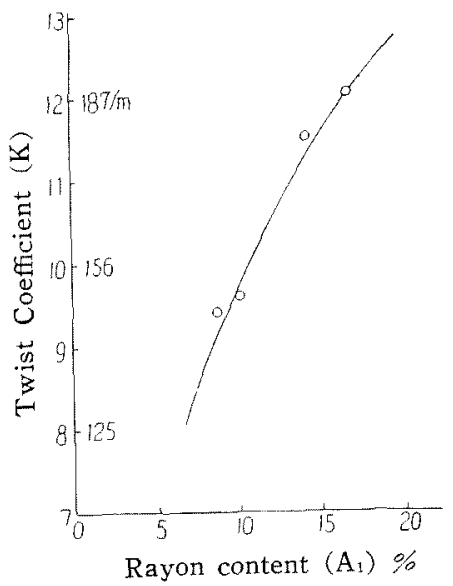

Fig. 3. Minimum twist when $\alpha=1$. 
to see what minimum degree of twist would be needed for $\alpha$ to equal one. The results of the measurement are given in Figure 3. The minimum twist needed for $\alpha=1$ in a yarn of $10 \sim$ $20 \%$ viscose rayon content is $K=8 \sim 12$. This twists is also expressible thus:

$$
M \cdot r=0.056 \sim 0.084
$$

where $r$ is the yarn diameter and $M$ the number of twists.

The absolute maximum number of twists which can be given to a yarn being $M \cdot r=0.13$ $\sim 0.14[5]$, a twist yarn in which $\alpha=1$ is a somewhat strongly twisted yarn.

\section{Experiment on the Rupture of Cord by Flexing Fatigue}

A bending fatigue tester governed by the same principle as Goodrich Flexing Tester [6] for tire-cord was used. Fine filaments $(110 \sim 120$ den.) of nylon, rayon and terylene of the properties shown in Table 2 were twisted and made into nylon, rayon and terylene cords, respectively, and the $\mathrm{S}-\mathrm{N}$ diagram of each kind of cord drawn as shown in Figure 4. Because the distribution of the fatigue life of filaments under various degrees of stress was logarythmic normal, the fatigue curve may be represented by mean of logarythm of fatigue life, i.e., the geometrical mean of fatigue life. The coefficient of correlation between stress and the mean of logarythm of fatigue life is about 0.9. Each curve in Figure 4 is explained thus:

Table 2. Tensile Properties of Tested Cords

\begin{tabular}{cccc}
\hline Cords & Den. & $\begin{array}{c}\text { Tensile strength } \\
(\mathrm{g} / \mathrm{den})\end{array}$ & $\begin{array}{c}\text { Elongation-to } \\
\text {-rupture } \\
(\%)\end{array}$ \\
\hline Rayon & $840 / 2$ & 1.17 & 27.8 \\
Terylen & $750 / 2$ & 4.43 & 23.9 \\
Nylon & $770 / 2$ & 5.59 & 31.2 \\
\hline \multicolumn{1}{c}{ Note: } & Ply twist & $(\mathrm{S}) 4 / \mathrm{cm}$ & \\
& Yarn twist & $(\mathrm{Z}) 4 / \mathrm{cm}$ &
\end{tabular}

$$
\begin{array}{ll}
\text { Nylon } & \sigma=3.74-0.48 \log N \\
\text { Terylene } & \sigma=3.81-0.69 \log N \\
\text { Rayon } & \sigma=1.19-0.21 \log N
\end{array}
$$

where, $\sigma$ is tensile stress ( $\mathrm{g} / \mathrm{den}) ; N$, half the number of repetitions of the load until rupture.

The same measurement was made of the following mixed twisted cords:

\section{(A) Rayon-Terylene Cord}

The fatigue lives of several rayon-terylene cords differing in the proportions of mixing (Table 3) were measured with the results given in Figure 5. Most of the dots therein make straight lines. On the whole, these lines gather slightly toward terylene.

\section{(B) Terylene-Nylon Cord}

The sample cords used are shown in Table 4 and the results of the measurement given

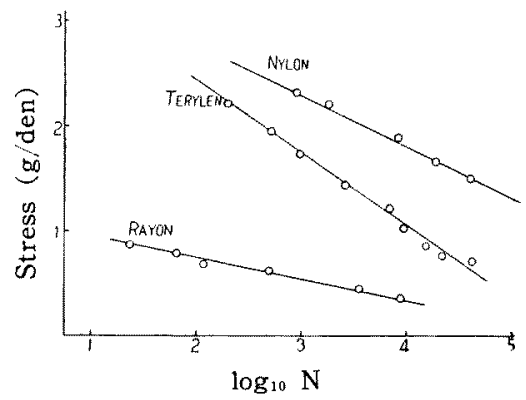

Fig. 4. S-N diagrams of rayon, terylene and nylon cords.

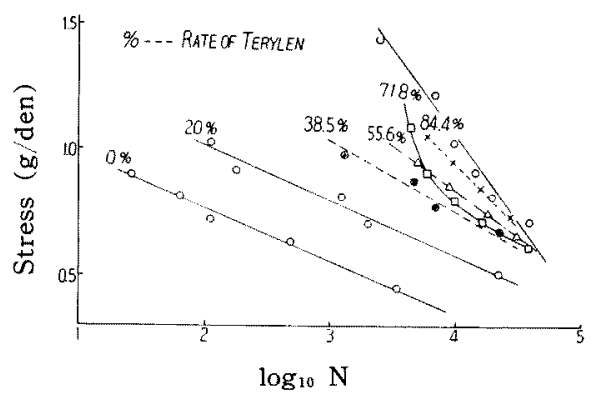

Fig. 5. S-N diagrams of blended cords made of rayon and terylene.

Table 3. Blended Cords Made of Rayon and Terylene.

\begin{tabular}{ccccccc}
\hline $\begin{array}{c}\text { Blended cords } \\
\text { (den.) }\end{array}$ & $\begin{array}{c}\text { Rayon } \\
\text { (den.) }\end{array}$ & $\begin{array}{c}\text { Terylene } \\
\text { (den.) }\end{array}$ & $\begin{array}{c}\text { Rayon } \\
\%\end{array}$ & $\begin{array}{c}\text { Terylen } \\
\%\end{array}$ & $\begin{array}{c}\text { Tensile strength } \\
\text { (g/den.) }\end{array}$ & $\begin{array}{c}\text { Elongation-to-rupture } \\
(\%)\end{array}$ \\
$720 / 2$ & 240 & 1200 & 16.7 & -33.3 & 3.11 & 24.8 \\
$840 / 2$ & 480 & 1200 & 28.6 & 71.4 & 3.43 & 27.4 \\
$810 / 2$ & 720 & 900 & 44.4 & 55.6 & 2.95 & 26.3 \\
$780 / 2$ & 960 & 600 & 61.5 & 38.5 & 2.22 & 25.8 \\
$750 / 2$ & 1200 & 300 & 80.0 & 20.0 & 1.80 & 25.2 \\
\hline
\end{tabular}

Note: Ply twist (S) $4 / \mathrm{cm}$, Yarn twist (Z) $4 / \mathrm{cm}$ 
in Figures 6 and 7 . The $\mathrm{S}-\mathrm{N}$ diagram in Fig ure 6 gather toward terylene, with hardly any change in their position even with an increase in the ratio of nylon which has a longer fatigue life. Figure 7 suggests that a cord of a nylon content of about $40 \%$ is shorter in fatigue life than an all-terylene cord. In other words, fatigue life decreases by the addition of filaments having a long life.

\section{(C) Nylon-Rayon Cord}

The sample cords used are described in Table 5. It is difficult to test nylon-rayon cords with the use of many different loads because nylon and rayon differ greatly in fatigue life. Accordingly, the fatigue life of each cord was measured with only one kind of load. The results are given in Figure 8, in which $\log N$ is shown in vertical lines and the mixing ratio in horizontal line.

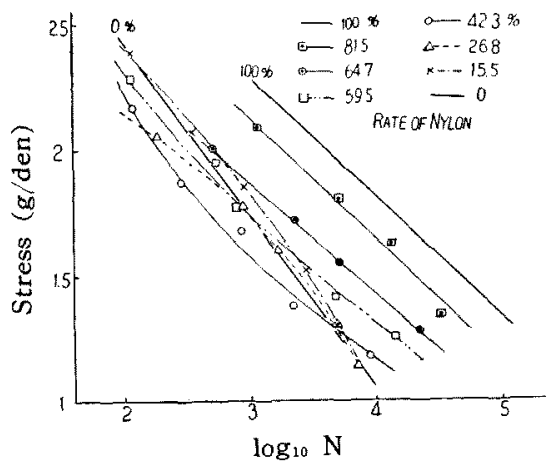

Fig. 6. S-N diagrams of blended cords made of terylene and nylon.

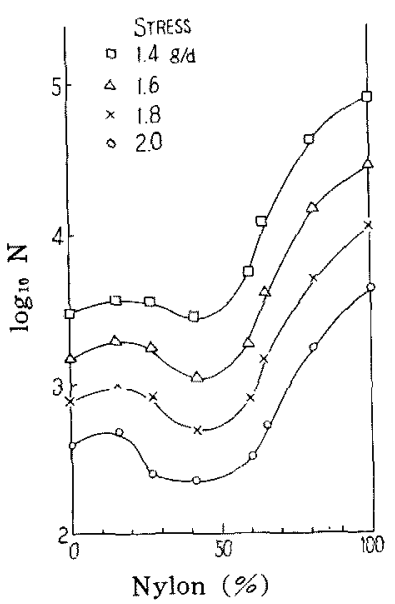

Fig. 7. Relation between $\log \mathrm{N}$ and rate of fibers in blended cords made of nylon and terylene.

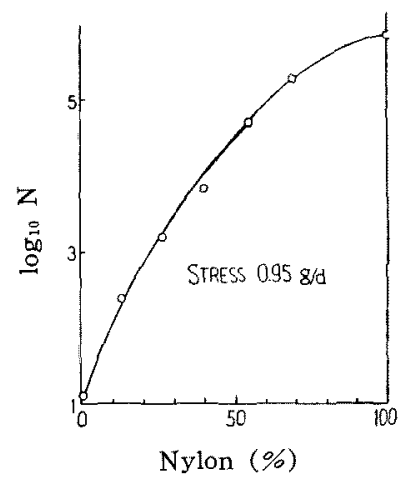

Fig. 8. Relation between $\log \mathrm{N}$ and rate of fibers in blended cords made of nylon and rayon.

Table 4. Blended Cords Made of Terylene and Nylon

\begin{tabular}{ccccccc}
\hline $\begin{array}{c}\text { Blended cords } \\
\text { (den.) }\end{array}$ & $\begin{array}{c}\text { Terylene } \\
\text { (den.) }\end{array}$ & $\begin{array}{c}\text { Nylon } \\
\text { (den.) }\end{array}$ & $\begin{array}{c}\text { Terylene } \\
(\%)\end{array}$ & $\begin{array}{c}\text { Nylon } \\
(\%)\end{array}$ & $\begin{array}{c}\text { Tensile strongth } \\
\text { (g/den.) }\end{array}$ & $\begin{array}{c}\text { Elongation-to-rupture } \\
(\%)\end{array}$ \\
\hline $810 / 2$ & 300 & 1320 & 18.5 & 81.5 & 4.41 & 27.7 \\
$850 / 2$ & 600 & 1100 & 35.3 & 64.7 & 4.32 & 25.4 \\
$740 / 2$ & 600 & 880 & 40.5 & 59.5 & 4.32 & 26.5 \\
$780 / 2$ & 900 & 660 & 57.5 & 42.3 & 4.29 & 25.7 \\
$820 / 2$ & 1200 & 440 & 73.2 & 26.8 & 4.36 & 23.7 \\
$710 / 2$ & 1200 & 220 & 84.5 & 15.5 & 4.28 & 22.4 \\
\hline
\end{tabular}

Note: Ply twist (S) $4 / \mathrm{cm}$, yarn twist (Z) $4 / \mathrm{cm}$

Table 5. Blended Cords Made of Nylon and Rayon.

\begin{tabular}{crccccc}
\hline $\begin{array}{c}\text { Blended cords } \\
\text { (den.) }\end{array}$ & $\begin{array}{c}\text { Nylon } \\
\text { (den.) }\end{array}$ & $\begin{array}{c}\text { Rayon } \\
\text { (den.) }\end{array}$ & $\begin{array}{c}\text { Nylon } \\
(\%)\end{array}$ & $\begin{array}{c}\text { Rayon } \\
(\%)\end{array}$ & $\begin{array}{c}\text { Tensile strength } \\
\text { (g/den.) }\end{array}$ & $\begin{array}{c}\text { Elongation-to-rupture } \\
(\%)\end{array}$ \\
\hline $830 / 2$ & 220 & 1440 & 13.3 & 86.7 & 1.44 & 26.7 \\
$820 / 2$ & 440 & 1200 & 26.8 & 73.2 & 1.88 & 27.4 \\
$810 / 2$ & 660 & 960 & 40.8 & 59.2 & 2.39 & 28.4 \\
$800 / 2$ & 880 & 720 & 55.0 & 45.0 & 2.70 & 31.0 \\
$790 / 2$ & 1100 & 480 & 69.6 & 30.4 & 3.20 & \\
\hline
\end{tabular}

Note: Ply twist (S) $4 / \mathrm{cm}$, yarn twist (Z) $4 / \mathrm{cm}$ 


\section{Theoretical Investigation of Rupture of Cord by Flexing Fatigue}

\subsection{Theoretical Estimaion of Fatigue Life}

For the sake of simplification, we assume that fatigue life is definite in the same kind of filament. Since blended cords of the same degree of twist differ only negligibly in their effects on fatigue life, it is enough to inquire only into the inbalance of stress between their constituent filaments.

Assume the blending of two kinds of filaments ( $A$ and $B$ ) having fatigue curves such as are given in Figure 9. Assume that $n_{a_{1}}$ is the fatigue life of filament $\mathrm{A}$ under stress $\sigma_{a}$ and $N_{b_{1}}$ the fatigue life of filament $B$ under $\sigma_{b} . \quad \sigma_{a}$ and $\sigma_{b}$ are assumed stress when time $t=0$. After the fatigue rupture of filament $A$, only filament $B$ gets loaded and stress increases to $\sigma_{b_{2}}$. Assume the fatigue life of filament $B$ under $\sigma_{b_{2}}$ to be $N_{b_{2}}$ and the repetition of loading from $n_{a_{1}}$ until the breakage of the whole cord to be $n_{b 2}$. Filament $B$ being breakable by multiple repeated stress on two levels, the following equation concerning a previous investigation ( 7 ) by the authors holds good here:

$$
\frac{n_{a_{1}}}{N_{b_{1}}}+\frac{n_{b_{2}}}{N_{b_{2}}}=b
$$

where $b$ is constant (usually nearly equal to one)

Accordingly, the fatigue life $N$ of the blended cord made of filaments $A$ and $B$ is given by the follwoing:

$$
N=n_{a_{1}}+n_{b_{2}}=n_{a_{1}}+N_{b_{2}}\left(b-\frac{n_{a_{1}}}{N_{b_{1}}}\right)
$$

The stress of both filaments, however, changes with time because the two filaments usually differ in the creep state. Assume the mechanical model of the blended cord to be three-element models joined together side by side (as in Fig. 10) and

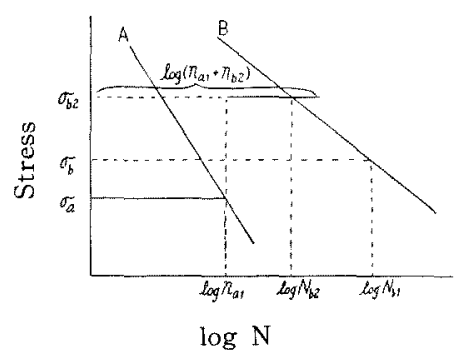

Fig. 9. Illustration for S-N diagrams of two kinds of fibers in a blended cord, and fatigue rupture of the fibers under multiple repeated stress at two stress levels. that their parameters are definable as follows:

$E_{a}, E^{\prime}{ }_{a}$ : Elastic coefficients of two springs of filament $A$.

$E_{b}, E_{b}^{\prime}$ : Elastic coefficient of two springs of filament $B$.

$\eta_{a}: \quad$ Viscosity coefficient of filament $A$.

$\eta_{b}:$ Viscosity coefficient of filament $B$.

On these assumptions we may express $\sigma_{a t}$ and $\sigma_{b t}$, the stresses of filaments $A$ and $B$, as a function of time, thus:

$$
\begin{aligned}
\sigma_{a t}= & {\left[\left\{E_{b}\left(1-e^{-t / \lambda b}\right)+E^{\prime}{ }_{b}\right\} E_{a} E_{a}^{\prime} \cdot \sigma\right] /\left[A _ { 1 } \left\{E_{b}(1\right.\right.} \\
& \left.\left.-e^{-t / \lambda b}\right)+E^{\prime}{ }_{b}\right\} E_{a} E^{\prime}{ }_{a}+B_{1}\left\{E_{a}\left(1-e^{-t / \lambda a}\right)\right. \\
& \left.\left.+E_{a}^{\prime}\right\} E_{b} \cdot E^{\prime}{ }_{b}\right] \\
\sigma_{b t}= & {\left[\left\{E_{a}\left(1-e^{-t / \lambda a}\right)+E^{\prime}{ }_{a}\right\} E_{b} E_{b}^{\prime} \cdot \sigma\right] /\left[A _ { 1 } \left\{E_{b}(1\right.\right.} \\
& \left.\left.-e^{-t / \lambda b}\right)+E^{\prime}{ }_{b}\right\} E_{a} E^{\prime}{ }_{a}+B_{1}\left\{E_{a}\left(1-e^{-t / \lambda a}\right)\right. \\
& \left.\left.+E^{\prime}{ }_{a}\right\} E_{b} \cdot E^{\prime}{ }_{b}\right]
\end{aligned}
$$

where $A_{1}$ and $B_{1}$ are the fractions of both filaments, $(A+B=1)$, and $\lambda_{a}=\eta_{a} / E_{a}^{\prime}, \lambda_{b}=\eta_{b} / E^{\prime}{ }_{b}$.

With the fatigue curves expressed as

$$
\left.\begin{array}{l}
A ; \sigma=M_{a}-m_{a} \log N \\
B ; \sigma=M_{b}-m_{b} \log N
\end{array}\right\}
$$

$M_{a}, M_{b}, m_{a}$ and $m_{b}$ are constant.

The following two equations are derivable on the fatigue lives $N_{A}$ and $N_{B}$, respectively,

$$
\left.\begin{array}{rl}
N_{A}^{-1}= & 10^{-N a / m a} \exp \left[\frac{f}{m_{a}}\right. \\
& \left.\times \frac{\left\{E_{b}\left(1-e^{-t / \lambda \Delta}\right)+E_{b}^{\prime} E_{a} E_{a}^{\prime}\right.}{H}\right] \\
N_{B}^{-1}= & 10^{-M s^{\prime} / m h} \exp \left[\frac{f}{m_{b}}\right. \\
& \left.\times \frac{\left\{E_{a}\left(1-e^{-t / \lambda a}\right)+E_{a}^{\prime}\right\} E_{b} E_{b}^{\prime}}{H}\right]
\end{array}\right\}
$$

where $f=\sigma \ln 10$ and $H$ is the dominator of equation (16).

This equation shows that the filaments break if bent with frequencies equalling $N_{A}$ or $N_{B}$

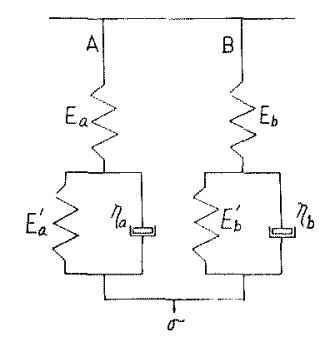

Fig. 10. Mechanical model of a blended cord. 
under stress of the degree which is produced $t$ minutes after the beginning of bending.

Frequent bending may be considered continuous. Therefore, the following equation is obtained by replacing $t$ in equation (18) with $\mathrm{n}$ :

$$
e^{t \cdot \lambda u}=e^{n / k \lambda \lambda}
$$

where $k$ : frequency of bending per unit time ( $k=4.4 / \mathrm{sec}$ in the present experiment.)

$n$ : Frequency of bending in $t$ minutes $(n=k t)$. $N_{A}^{-1}$ and $N_{B}^{-1}$, then, are functions of $n$ :

$$
N_{A}^{-1}=f_{a}(n), N_{B}^{-1}=f_{b}(n)
$$

It is assumed that filament $A$ is broken by bending of frequency $n_{a 1}$ under the stress given in equation (16). Divide $n_{a_{1}}$ equally into parts $h$ in number, assume bending of frequency $n_{h}$ in each part and assume the law of fatigue rupture by multiple repeated stress on two levels. Then:

$$
n_{n}+\frac{n_{h}}{N_{2}}+\cdots+{ }_{N_{h}}^{n_{h}}=a
$$

where $a$ is constant (usually nearly equal to one).

$h$ being assumed to be infinitely large, the following equation develops:

$$
\int_{0}^{n a 1} f_{a}(n) d n=a
$$

The value of $n_{a 1}$ is calculable by integrating equation (20). This integration is, however,

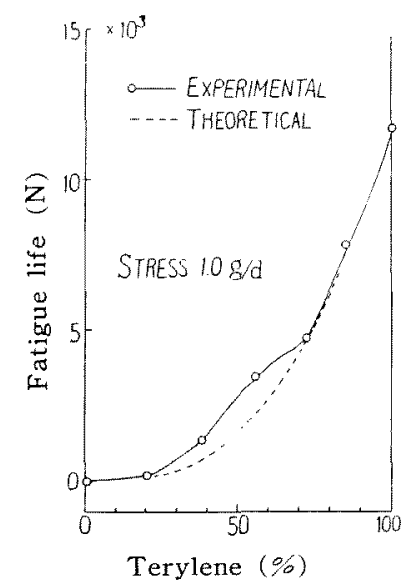

Fig. 11. Comparison of experimental and theoretical results on the fatitgue life of blended cords made of rayon and terylene.

Table 6. Partmeters of Mechanical Model

\begin{tabular}{crrc} 
& Nylon & Terylene & Rayon \\
$E_{a} \cdot \mathrm{g} / \mathrm{den}$ & 12.7 & 38.0 & 12.1 \\
$E_{a}^{\prime} \cdot \mathrm{g} / \mathrm{den}$. & 35.8 & 101.6 & 6.43 \\
$\lambda_{a}=\eta_{a} E^{\prime} a$ & $505 \mathrm{sec}$ & $351 \mathrm{sec}$ & $471 \mathrm{sec}$ \\
\hline
\end{tabular}

irresolvable, but it is calculable graphically from the $N^{-1}-n$ curve of equation (19). The fatigue life of the blended cord under discussion is derivable by using $n_{a 1}$ as folows: Assume the number of repetitions from $n_{i 1}$ until the breaking of the whole cord to be $n_{b 2}$.

$$
\int_{0}^{n a 1} f_{b}(n) d n+\frac{n_{b 2}}{N_{b 2}}=b
$$

The fatigue life $\mathrm{N}$ of the blended cord is, then, given as follows:

$$
N=n_{\llcorner 1}+n_{b_{2}}=n_{a_{1}}+N_{b_{2}}\left\{b-\int_{0}^{n a 1} f_{b}(n) d n\right\}
$$

$N_{b 2}$ is derived from equation (17).

$$
N_{b 2}=10^{\left(M_{\left.b-\left(\sigma / B_{1}\right)\right\}}\right) \mathrm{mo}}
$$

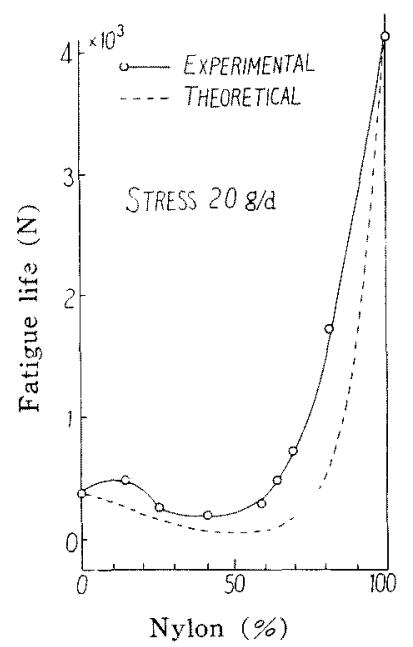

Fig. 12. Comparison of experimental and theoretical results on the fatigue life of blended cords made of terylene and nylon.

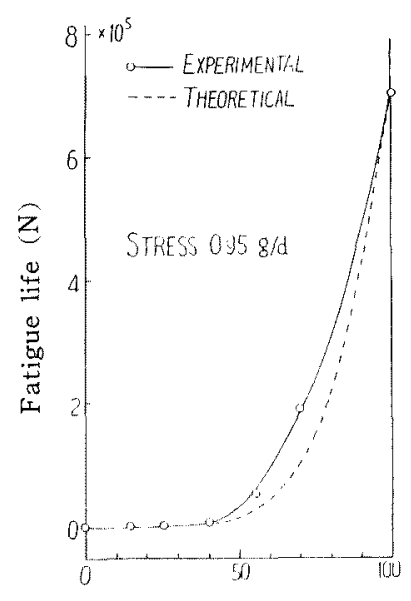

Nylon $(96)$

Fig. 13. Comparison of experimental and theoretical results on the fatigue life of blended cords made of nylon and rayon. 


\section{3-2. Comparing Theory and Experiment}

Curves showing the ratios of mixing to the logarythm of fatigue life are found to shift parallel with varying degrees of stress. Accordingly, here we will take up one degree of stress.

In Figures 11, 12 and 13 measured values are compared with the theoretical values of blended cords of terylene-rayon, nylon-terylene and nylon-rayon. Their fatigue lives are shown in vertical lines in the figures. The theoretical values were graphically calculated by equaion (22), $a$ and $b$ being assumed to be equal to one. The parameters in Figure 10, approximately calculated by the creep method, are listed in Table 6 . The theory and the measurement agree fairly well.

\section{Best Conditions for Blending}

Measured under stress $\sigma$, filament $A$ is shorter in fatigue life than $B$. Generally, there is the following relation between stresses $\sigma_{v}$ and $\sigma_{b}$ at $t=0$ :

$$
\sigma=A_{1} \sigma_{a}+B_{1} \sigma_{b}
$$

and the longer the time that passes, the closer these stresses get about lineally to $\sigma_{a}{ }^{\prime}$ and $\sigma_{b}{ }^{\prime}$, namely, stresses after the passsge of a long time. Therefore, if the $N^{-1}$-n curve of one filament is convex, that of the other is concave. The stress of each fiber is divisible into the six groups, (I) (VI), shown in Figure 14. One of the two kinds of filaments which satisfies the equation $\int_{0}^{n} N^{-1} d n=1$ first breaks after bending with frequency $\mathrm{n}$. After bending with the same frequency, filaments of the other kind increase abruptly in stress and decrease sharply in fatigue life.

On the other hand, if both filaments break at the same time, they reach fatigue life with less
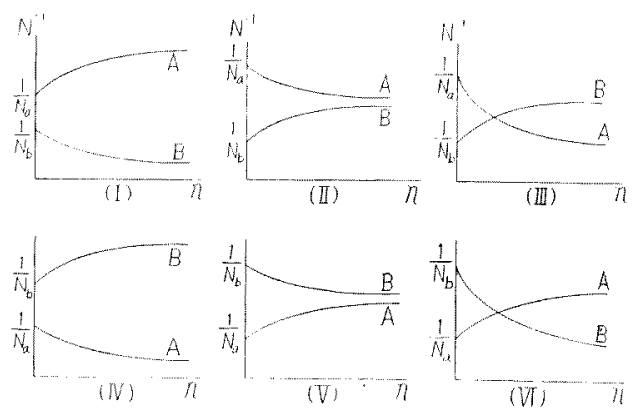

(1) $)$ (III) $N_{b}>N_{i d}$, (W) $-($ (V) $) N_{a}>N_{b}$

Fig. 14. $\mathrm{N}^{-1}-n$ curves classified. stress than if they break at differerent times. The simultaneous breakage of two different filaments is the best condition for their blending, provided the total value of ratios of repetitions of the load is equal to one. This best condition is expressed as follows:

$$
\int_{u}^{n} f_{n}(n) d n=\int_{u}^{n} f_{L}(n) d n=1
$$

and arises only in cases (III) and (IV) in Figure 14.

In those cases, the filament which has a longer fatigue life at the initial stress increases in stress with time (i. e., it becomes difficult to creep). The filament which has a shorter fatigue life at the initial stress decreases in stress with time (i. e., it becomes easy to creep). If the fatigue curves and the parameters of the mechanical models of the two filaments are known, the best ratio of mixing them can be calculated by equation (23).

The best filament for blending with other filaments is one which satisfies equation (23), irrespective of the mixing ratio and the degree of stress.

The authors have sought to discover what fatigue curve the ideal fiber should have and what parameters its mechanical model should have. Assume fatigue lives $N_{u}$ and $N_{b}$ of two filaments under initial stresses $\sigma_{u}$ and $\sigma_{b}$. Then, $N_{a}$ and $N_{b}$ must be equal and are expresssd as follows:

$$
N_{a}=10^{\left(M r a-\sigma_{a}\right) / m a}, N_{b}=10^{\left(M_{b} b-\sigma_{b}\right) / m b}
$$

Therefore:

$$
\begin{gathered}
\left(M_{u}-\sigma_{a}\right) / m_{a}=\left(M_{b}-\sigma_{b}\right) / m_{b} \\
\therefore \quad M_{a} m_{b}-M_{b} m_{a}+\sigma_{b} m_{a}-\sigma_{a} m_{b}=0 \\
M_{a} m_{b}-M_{b} m_{a}+E_{b} m_{a} \sigma \\
A_{1} E_{a}+B_{1} E_{b} \\
\quad-\frac{E_{a} m_{b} \sigma}{A_{1} E_{a}+B_{1} E_{b}}=0 \\
\therefore \quad\left(A_{1} E_{u}+B_{1} E_{b}\right)\left(M_{a} m_{b}-M_{b} m_{a}\right) \\
\quad+\sigma\left(E_{b} m_{a}-E_{a} m_{b}\right)=0 \quad \text { (See eq. 16) }
\end{gathered}
$$

To satisfy the above equation no matter what the values $A_{1}, B_{1}$ and $\sigma$ are, there must be the following equation:

$$
M_{a} m_{b}=M_{b} m_{\alpha} \text { and } E_{b} m_{a}=E_{a} m_{b}
$$

For equation (24) to be satisfied, both straight lines must cross each other on the horizontal line $\sigma=0$. (See Fig. 15). In order that $N_{a}=N_{b}$ holds even after the passage of time, there must be the following equation:

$$
\sigma_{a t}=\sigma_{a t}=\sigma_{a}^{\prime} \text { or } \sigma_{b}^{\prime}=\sigma_{b t}=\sigma_{b}^{\prime}
$$


The condition requisite to $\sigma_{a}=\sigma_{a}^{\prime}$ is as follows, calculated from changes in the stress of the mechanical model:

$$
E^{\prime}{ }_{a} E_{b}=E_{a} E^{\prime}{ }_{b}
$$

Moreover, satisfaction of $\sigma_{a}=\sigma_{\imath, \iota}$ needs the following equation derived from equation (16):

$$
\lambda_{a}=\lambda_{b} \quad \therefore \eta_{a} / E_{a}^{\prime}=\eta_{t} / E_{b}^{\prime}
$$

Equations $(24) \sim(26)$ lead to this further equation:

$$
\frac{M_{a}}{M_{b}}=\frac{m_{a}}{m_{b}}=\frac{E_{b}}{E_{a}}=\frac{E^{\prime}{ }_{b}}{E^{\prime}{ }_{a}}=\frac{\eta_{b}}{\eta_{a}}
$$

An ideal blended cord of filaments $A$ and $B$ satisfies equation (27). The first two terms of this equation represent the condition of fatigue life; the other three, the mechanical properties of the two filaments. The fatigue life of this ideal cord is easily calculable if the fatigue curve of both filaments is known. Calculation shows that even this ideal cord is shorter in fatigue life than the arithmetical mean of the fatigue lives of the component filaments.

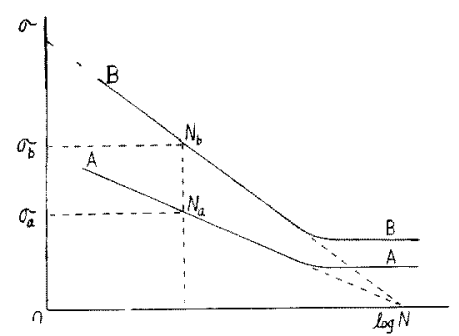

Fig. 15. S-N diagrams of ideal fibers.

\section{Conclusions}

The tensile strength and the fatigue life of even an ideally blended yarn or cord are inferior to the arithmetical mean of these properites of the component filaments, although, in some cases, an ideally blended yarn or cord is strong enough to require, for its tensile rupture, work energy which exceeds the arithmetical mean of the work energy of the component filaments. Blending is, therefore, not always to be recommended for purposes which require mechanical strength. New methods of blending must be devised which give enough other advantages to compensate for the weakness of mechanical strength.

The best condition for blending where tensile rupture is the aim is that the content of the filament of smaller breaking elongation be comparatively small and that the yarn be twisted in a degree over half the maximum number of twists.

The best condition for blending where bending fatigue is the aim is that the relation between the fatigue curve and the parameters of the mechanical model closely resemble the relation illustrated in equation (27). However, filaments meeting this condition are scarce.

Filaments of a kind having a long fatigue life and difficult to creep, if blended with filaments of another kind which is short in fatigue life and easy to creep, may possibly give a good cord.

\section{Literature cited}

(1) Reports on fiber blends at Annual Conference of Textile Institute. J. Text. Inst., Vol. 43, No. 8, p. 347 (1952)

(2) H. Noshi; J. Soc. Text. Cellulose Ind. Japan, Japanese edition, Vol. 11, No. 12 , p. 812 (1955)

(3) S. Yamaguchi, et al.; J. Text. Mach. Soc., Japanese edition. Vol. 10, No. 12, p.935 (1957)

(4) K. Fujino; Mechanical Properties of Yarns, Boshoku Zasshisha, Osaka, Japan

(5) K. Fujino and H. Noshi; J. Text. Mach. Soc., Japanese edition, Vol. 4, No. 7, p. 385 (1951)

[6] C.B. Budd; Text. Res. J., Vol. 21. No. 3, p. 174 (1951)

(7) H. Noshi; No published 